\title{
ESTABILIZAÇÃO DA FORMA ABERTA DE LECITASE ATRAVÉS DA MODIFICAÇÃO FÍSICA COM POLÍMEROS IÔNICOS
}

\author{
J.C.S. DOS SANTOS ${ }^{1,2}$, C.GARCIA-GALÁN ${ }^{1}$, R. C. RODRIGUES ${ }^{3}$,H. B. SANT'ANA ${ }^{2}$, L. R. B. \\ GONÇALVES ${ }^{2}$, ROBERTO FERNÁNDEZ-LAFUENTE ${ }^{1}$ \\ ${ }^{1}$ Universidad Autónoma de Madrid. Instituto de Catálisis y Petroleoquímica-CSIC. \\ ${ }^{2}$ Universidade Federal do Ceará, Departamento de Engenharia Química. \\ ${ }^{3}$ Universidade Federal do Rio Grande do Sul, Instituto de Ciência e Tecnologia dos Alimentos. \\ E-mail para contato: jscleiton@gmail.com
}

\begin{abstract}
RESUMO - Lecitase Ultra é uma fosfolipase A1, uma enzima que foi imobilizada covalentemente em brometo de cianogênio-agarose (CNBr), mantendo $70 \%$ da atividade inicial. A atividade da enzima imobilizada, Lecitase-CNBr, na presença de detergentes, tais como Triton X-100, dodecil sulfato de sódio (SDS), cetiltrimetil brometo de amônio (CTAB) foi melhorada em cerca de até $800 \%$ quando utilizado CTAB. No entanto, CTAB e Triton-X100 apresentaram um efeito negativo sobre a estabilidade da enzima, mesmo quando foram usados em baixas concentrações $(0,01 \%(\mathrm{v} / \mathrm{v}))$, e nem o detergente SDS pode ser usado por longos períodos na concentração de $1 \%$. O revestimento da enzima imobilizada com polietilenimina (PEI), em tampão aquoso, produziu um aumento de 3 vezes na atividade da enzima. No entanto, na presença de SDS, a 0,1\% (v/v), este revestimento produziu um aumento de 50 vezes na atividade da enzima. Usando PEI e $0,01 \%$ (v/v) de CTAB, a atividade Lecitase diminuiu em $10 \%$.
\end{abstract}

\section{INTRODUÇÃO}

O melhoramento da atividade enzimática é um dos principais focos no desenvolvimento de novos biocatalisadores. Este aumento de atividade é conseguido pela modificação da enzima utilizando diversas ferramentas, tais como: mutagênese dirigida, evolução dirigida, modificação química, dentre outros (SANTOS et al. 2014b).

O revestimento de enzimas em fase sólida com polímeros iônicos é um método bastante simples e rápido para o melhoramento da atividade de enzimas, como temos referencia nos trabalhos de Garcia-Galan et al. (2014) e Santos et al. (2014 a,b).

Lecitase Ultra é uma fosfolipase A1 artificial. Uma enzima que tem sido obtida a partir da fusão dos genes da lipase de Thermomyces lanuginosus, como forma de obter uma boa estabilidade, e a fosfolipase de Fusarium oxysporum, objetivando uma atividade de fosfolipase.

Em alguns aspectos, Lecitase Ultra comporta-se como uma enzima lipase padrão, apresentando uma capacidade de se tornar adsorvida em superfícies hidrofóbicas em baixa força iônica, segundo De Maria et al. (2007) e Fernandez-Lorente et al. (2007). Assim, no presente trabalho, buscamos obter a 
estabilização da conformação aberta de Lecitase, através da modificação física, com polímeros iônicos, após ativação com detergentes.

\section{MATERIAIS E MÉTODOS}

A Lecitase foi doada da Novozymes (Espanha) (16 mg de proteína / mL ter um pNPB atividade de 5,6 U / mg de proteína). O Brometo de cianogênio reticulado 4\% agarose (CNBr) da $G E$ Healthcare (Pollards Wood, UK). A Polietilenimina (ramificada, Mn 10000, Mw 25000 Da), o sulfato de dextrano (DS, média Mw, 9000 - 20000 Da), o butirato de p-nitrofenilo (pNPB), o Triton $\mathrm{X}-100$, dodecil sulfato de sódio (SDS), o brometo de cetiltrimetilamónio (CTAB) e o éter pnitrofenilfosfato (D-pNPP) da Sigma Chemical Co. (St. Louis, MO, EUA).

\subsection{Determinação da atividade da enzima}

Este ensaio foi realizado através da medição do aumento da absorbância, a $348 \mathrm{~nm}$, produzido pela liberação de p-nitrofenol na hidrólise de $0,4 \mathrm{mM}$ de p-nitrophenylbutyrate (p-NPB), em fosfato de sódio $100 \mathrm{mM}$, a pH 7,0 e $25{ }^{\circ} \mathrm{C}$ (sob estas $\varepsilon$ condições é $5150 \mathrm{M}^{-1} . \mathrm{cm}^{-1}$ ). Uma unidade internacional de atividade $(\mathrm{U})$ foi definida como a quantidade de enzima que hidrolisa $1 \mu \mathrm{mol}$ de $\mathrm{p}$ NPB por minuto, sob as condições descritas anteriormente. A concentração de proteína foi determinada utilizando o método de Bradford (1976), e a de albumina de soro bovino foi utilizada como referência.

\subsection{Imobilização de Lecitase em $\mathrm{CNBr}$-agarose}

Um volume de $2,8 \mathrm{ml}$ de Lecitase comercial foi diluída em $67,5 \mathrm{~mL}$ de fosfato de sódio $5 \mathrm{mM}$, contendo $0,05 \%(\mathrm{~m} / \mathrm{v})$ de dodecil sulfato de sódio, a pH 7 e $4{ }^{\circ} \mathrm{C}$. Em seguida, foi adicionado $15 \mathrm{~g}$ de $\mathrm{CNBr}$ e a atividade de sobrenadante e suspensão foram seguidas usando p-NPB. A imobilização da enzima foi determinada por incubação do suporte com etanonamina $1 \mathrm{M}$, a pH 8 , durante $12 \mathrm{~h}$. Ao final, a preparação imobilizada foi lavada com água destilada abundante.

\subsection{Revestimento de Lecitase imobilizada com polímero iônico}

O protocolo foi seguido para o revestimento de Lecitase imobilizada por polímeros iônicos, como demostrado nos trabalhos de Santos et al. (2014 a). Foram adicionadas $10 \mathrm{~g}$ de Lecitase imobilizadas a $100 \mathrm{ml}$ de PEI, a um pH de 7 ou DS a pH $5(1 \mathrm{mg} / \mathrm{mL})$. Em alguns casos, a concentração de detergente foi adicionada 2 minutos antes de adicionar o polímero, mantendo-se esta mistura sob agitação suave, durante um período máximo de 24 horas. A atividade durante a incubação foi seguida pelo protocolo de atividade de p-NPB como descrito acima. 


\subsection{A análise do efeito dos detergentes sobre a estabilidade dos derivados de CNBr-Lecitase}

Para verificar a estabilidade dos derivados de enzima na presença de detergentes, $1 \mathrm{~g}$ de enzima imobilizada foi suspenso em $5 \mathrm{~mL}$ de $10 \mathrm{mM}$ de fosfato de sódio, a pH 7 a $25^{\circ} \mathrm{C}$. Periodicamente, foram retiradas amostras, e a atividade foi medida usando p-NPB. Uma suspensão de CNBr Lecitase, na ausência de detergente, foi usada como referencia. Foi considerado $100 \%$ do valor da atividade obtida, usando a suspensão de referência, e adicionando à mistura de reação a quantidade equivalente de detergente em cada amostra (para discriminar/ os efeitos inibitórios de ativação do detergente problema nas presentes suspensões).

\section{RESULTADOS}

\subsection{Efeito dos detergentes sobre a atividade enzimática}

Três detergentes com características diferentes foram utilizados para verificar o efeito na atividade enzimática, são eles: CTAB, um surfactante catiônico; SDS, um agente tensoativo aniônico; e Triton X-100, um surfactante não iônico. A Figura 1 nos mostra os efeitos sobre a atividade enzimática de cada detergente. No intervalo de concentrações estudadas, a preparação CNBr-Lecitase foi hiperativada na maioria dos casos. A maior hiperativação foi detectada quando utilizado CTAB (em quase 8 vezes), além disso, foi observado que o efeito aumentou junto com o aumento da concentração de detergente. Usando SDS, em baixas concentrações, foi obtido um efeito positivo sobre a atividade enzimática, ocorrendo assim um o aumento da atividade por duas vezes, quando foi utilizada uma concentração de $0,01 \%$ desse detergente. No entanto, a $0,1 \%$ de SDS, a atividade foi menor do que na ausência de SDS. Com Triton X-100, utilizando baixas concentrações, percebeu-se um pequeno efeito sobre a atividade da enzima. Contudo quando foi usada uma concentração de 0,1 $\%$ desse detergente, a atividade foi quase três vezes maior do que a atividade do derivado controle.

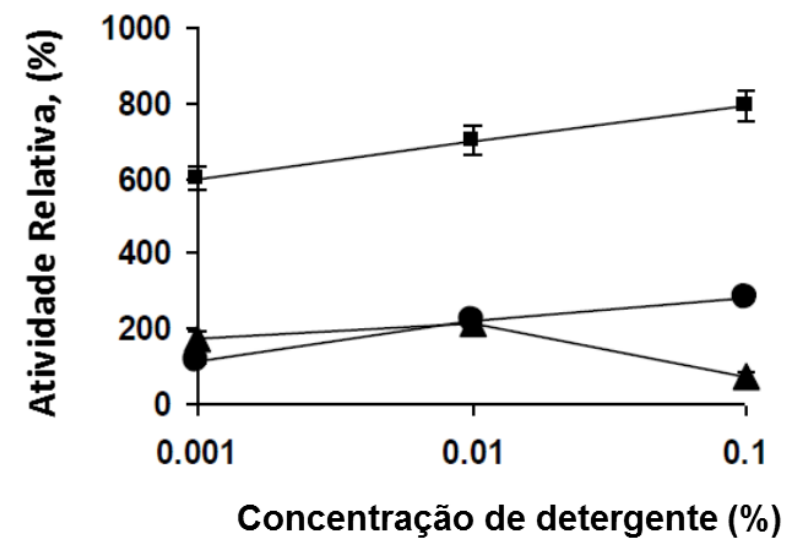

Figura 1 - Efeito dos detergentes na atividade da Lecitase imobilizada. Atividade foi determinada utilizando pNPB como indicado na seção de métodos.

Triângulos: SDS, quadrados: CTAB, Círculos: Triton X-100. 
Além disso, a preparação enzimática foi incubada na presença destes detergentes, objetivando verificar o seu efeito sobre a estabilidade da enzima (Tabela 1). Utilizando 0,1\% de SDS, a atividade enzimática observada, depois de 24 h, foi de quase $100 \%$, enquanto que, com $0,1 \%$ de Triton X-100, a atividade diminuiu lentamente, com CTAB, a atividade diminuiu rapidamente. A atividade recuperada, após 24 horas, foi de $80 \%$ utilizando Triton X-100, e 10\% usando CTAB. A atividade recuperada assim foi maior quando foi utilizada uma concentração de $0,01 \%$, para ambos os detergentes.

Tabela 1- Efeito da presença de detergentes na atividade de CNBr-Lecitase.

$\mathrm{CNBr}$-Lecitase foi incubado a pH 7 e $25^{\circ} \mathrm{C}$ em $25 \mathrm{mM}$ de fosfato de sódio, com concentração indicada de detergente, durante $24 \mathrm{~h}$. Em seguida, as preparações foram lavadas e a atividade do biocatalisador foi medida utilizando pNPB (ver métodos).

* 100 é a atividade inicial do biocatalisador.

\begin{tabular}{ccc}
\hline Detergente & Concentração $(\%),(\mathrm{v} / \mathrm{v})$ & Atividade recuperada $(\%)^{*}$ \\
\hline Ausente & - & 100 \\
Triton X-100 & 0.1 & $60 \pm 3$ \\
Triton X-100 & 0.01 & $80 \pm 3$ \\
SDS 0.1 & 0.1 & $100 \pm 5$ \\
SDS 0.01 & 0.01 & $100 \pm 4$ \\
CTAB & 0.1 & $>10$ \\
CTAB & 0.01 & $60 \pm 2$ \\
\hline
\end{tabular}

Embora, os três detergentes apresentassem efeitos positivos sobre a atividade da enzima, a presença destes na solução tinha efeito negativo sobre a estabilidade da enzima; o efeito foi mais significativo, fora com o detergente mais hiperativante (CTAB). Usando SDS, foi observado um maior efeito de hiperativação, quando utilizado uma concentração máxima, de até $0,1 \%$, desse detergente. No entanto, a concentração máxima que pode ser utilizada, por um período de tempo prolongado, fora de $0,01 \%$. Os efeitos positivos dos detergentes sobre a atividade da lipase é o mais provável, pelo menos parcialmente, devido à estabilização da conformação aberta da enzima, embora algumas outras alterações positivas na estrutura da enzima não possam ser desconsideradas. Os efeitos negativos poderiam incidir devido a uma distorção da estrutura da enzima, ou da inibição da atividade enzimática.

\subsection{Efeito da incubação da enzima imobilizada na presença de polímeros e detergentes.}

A preparação $\mathrm{CNBr}$-Lecitase foi incubada, em diferentes concentrações de detergentes, seguida pela adição de DS ou PEI para estabilizar a enzima modificada. A Figura 2 mostra a incubação da enzima imobilizada com diferentes concentrações de SDS. 


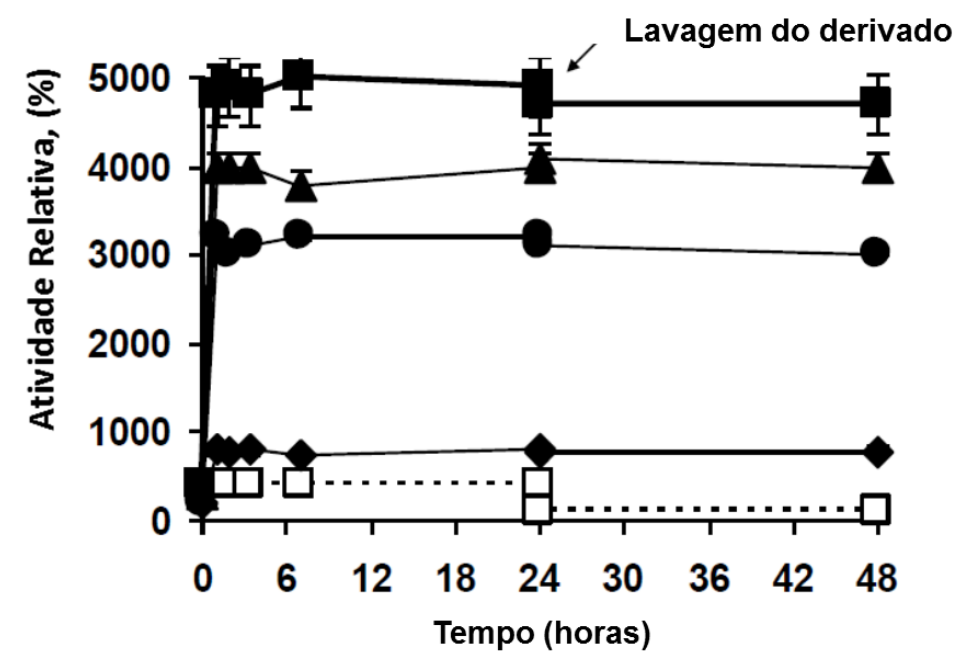

Figura 2. Efeito da concentração de SDS e do tempo de incubação sobre a atividade de

Lecitase imobilizada na presença ou ausência de PEI. Os experimentos foram realizados como descrito na secção de Métodos. PEI foi adicionado após 10 minutos de incubação com o detergente.

Linha tracejada, quadrados vazios: CNBr-Lecitase incubadas com 0,1\% de SDS, losango: $\mathrm{CNBr}$ Lecitase incubadas com 0,002\% de SDS e PEI, círculos: CNBr-Lecitase incubadas com 0,01\% SDS e PEI, Triângulos: CNBr Lecitase incubadas com 0,05\% de SDS e PEI, Quadrados: CNBr Lecitase incubadas com $0,1 \%$ SDS e PEI.

$\mathrm{Na}$ ausência de polímero, a adição de surfactante aumenta a atividade da enzima cerca de 2 vezes, e a atividade manteve-se praticamente inalterada após $24 \mathrm{~h}$ de incubação, com uma concentração máxima utilizada de SDS de 0,1\%. Depois da lavagem da enzima numa solução tampão, a atividade inicial foi recuperada, perdendo a "hiperativação", causada pela presença de detergente.

Quando PEI é adicionado à suspensão de enzima, depois de vários minutos de incubação em SDS, a atividade aumentou fortemente, e este aumento na atividade é mais elevado se compararmos quando aumentamos a concentração de SDS, onde após 30 minutos, a atividade permaneceu inalterada durante 24 horas. Enquanto o revestimento da enzima imobilizada com PEI, na ausência de detergente, induziu um aumento de 3 vezes na atividade, de acordo com o trabalho de Santos et al. (2014 a), a presença de 0,1\% de SDS antes da adição de PEI, melhorou a atividade enzimática em, aproximadamente, $45-50$ vezes.

Depois de lavar com água as preparações enzimáticas tratadas com os polímeros, a atividade da enzima imobilizada, revestida com PEI, na presença de SDS, manteve-se similarmente a níveis de hiperativação, mesmo após um mês de incubação a $25^{\circ} \mathrm{C}$, e a um $\mathrm{pH}$ de 7 . Já o revestimento de CNBr-Lecitase com DS levou a um decréscimo de, aproximadamente, $50 \%$ da atividade inicial na presença ou ausência de qualquer concentração de SDS, mesmo depois de lavar os derivados para eliminação do detergente (Tabela 2).

Usando 0,01 \% (v/v) de CTAB, na ausência de polímeros iônicos, a atividade diminuiu a 70\%, 
aproximadamente, após 24 h (Tabela 2). No entanto, foi observado que quando o PEI é adicionado à solução contendo CTAB, ocorre uma hiperativação inicial, semelhante à encontrada usando PEI na ausência de surfactantes (resultados não mostrados), mas, após $24 \mathrm{~h}$, a atividade foi menor que o CNBr-Lecitase sem polímeros. Posteriormente a lavagem, para eliminar o detergente, foi verificada uma diminuição da atividade em cerca de $10 \%$ (Tabela 2). Entretanto, ao usar DS, os resultados eram bem diferentes. Neste caso, o uso de $0,01 \%$ de CTAB causou uma hiperativação (150 \%), verificada depois de lavar as preparações (Tabela 2).

Tabela 2 - Atividade recuperada dos derivados de CNBr- Lecitase incubados em polímeros e /ou detergentes. O revestimento da enzima é descrito na seção de métodos. Atividade foi determinada utilizando pNPB como o substrato. $\mathrm{O}$ polímero foi adicionado à suspensão depois de incubação com o detergente. A atividade é dada como atividade relativa (100 corresponde a enzima não modificada) após $24 \mathrm{~h}$ de incubação no detergente e/ou polímero, lavagem com água e incubação a $25^{\circ} \mathrm{C}$ em tampão durante mais $24 \mathrm{~h}$.

\begin{tabular}{cccc}
\hline Detergente & Concentração $(\%)(\mathrm{v} / \mathrm{v})$ & Polímero & Atividade relativa \\
\hline Ausente & - & Ausente & $100 \pm 5$ \\
Ausente & - & PEI & $300 \pm 10$ \\
Ausente & - & DS & $50 \pm 2$ \\
SDS & 0,1 & Ausente & $100 \pm 5$ \\
SDS & 0,002 & PEI & $800 \pm 30$ \\
SDS & 0,01 & PEI & $3,000 \pm 100$ \\
SDS & 0,05 & PEI & $4,000 \pm 150$ \\
SDS & 0,1 & PEI & $5,000 \pm 150$ \\
SDS & 0,002 & DS & $50 \pm 3$ \\
SDS & 0,01 & DS & $45 \pm 2$ \\
SDS & 0,05 & DS & $55 \pm 2$ \\
SDS & 0,1 & DS & $50 \pm 2$ \\
CTAB & 0,01 & Ausente & $70 \pm 5$ \\
CTAB & 0,01 & PEI & $10 \pm 0.2$ \\
CTAB & 0,01 & DS & $150 \pm 5$ \\
Triton X-100 & 0,01 & Ausente & $85 \pm 5$ \\
Triton X-100 & 0,01 & PEI & $400 \pm 20$ \\
Triton X-100 & 0,01 & DS & $15 \pm 3$ \\
\hline
\end{tabular}

Usando $0,01 \%(\mathrm{v} / \mathrm{v})$ de Triton $\mathrm{X}$ - 100, na ausência de polímeros, a atividade diminuiu ligeiramente após 24 h (15-20 \%) (Tabela 2). Na presença de PEI, a atividade do biocatalisador foi 4 vezes mais elevada, um pouco maior do que quando foi utilizado PEI na ausência de surfactante (Tabela 2). Ao utilizar DS depois de incubar com Triton X - 100, foi observada uma atividade final de cerca de $15 \%$ menor, do que quando usando DS na ausência de detergente ( Tabela 2 ). 


\section{9 a 22 de outubro de 2014 \\ Florianópolis/SC}

Mesmo levando em conta a natureza iônica dos polímeros e dos detergentes, é difícil encontrar padrões para explicar os resultados. O SDS tem efeitos positivos, principalmente, com PEI, enquanto que o CTAB (que tem um efeito negativo sobre a estabilidade da enzima) apresenta certo efeito positivo sobre a atividade da enzima utilizando DS, o que é surpreendente, porque o DS tinha um efeito negativo sobre a atividade enzimática, e seria de esperar que favorecesse a acumulação de CTAB no ambiente com enzima.

Aparentemente, muitos fatores estão a contribuir simultaneamente para o sistema e, portanto, uma explicação simples destes resultados não pode ser dada; os efeitos individuais do detergente e polímeros em estrutura global da enzima podem ser muito difíceis de explicar e prever, como o seu efeito conjunto pode ser quase impossível de interpretar. No entanto, em alguns casos, certa hiperativação importante foi identificada.

Assim, utilizando-se p-NPB como substrato, o efeito mais positivo sobre a atividade da enzima se deu utilizando SDS, seguido por revestimento de PEI, produzindo um derivado de $\mathrm{CNBr}$ Lecitase, proposto cerca de 50 vezes mais ativo do que a preparação sem polímeros.

\section{CONCLUSÃO}

A atividade dos derivados de $\mathrm{CNBr}$-Lecitase pode ser aumentada pela incubação com baixas concentrações de detergentes, assim a atividade final é resultante de vários fatores, desde a estabilização da forma aberta da lipase à inibição da enzima, e mesmo à distorção. No entanto, em adição, complicações no uso industrial, detergentes demonstram alguns efeitos deletérios em propriedades da Lecitase, afetando, principalmente, a sua estabilidade. A incubação das preparações em soluções de detergente, junto ao revestimento da enzima com polímeros iônicos, nos permitiu obter preparações hiperativadas. No entanto, foi necessário selecionar o polímero e o detergente apropriado para maximizar os efeitos positivos, e essa seleção deve ser baseada em estudo empírico, devido à complexidade do processo envolvido. Além disso, espera-se que esta estratégia possa ser útil, para obtenção de preparações de lipase hiperativada de outras preparações de enzimas imobilizadas covalentemente.

\section{AGRADECIMENTOS}

Os autores agradecem ao apoio do Governo Espanhol e ao CNPq (Brasil), pelas bolsas de doutorado para Ms. García-Galán (Governo Espanhol) e o Sr. dos Santos (CNPq, Brasil). Os autores gostariam de agradecer ao Sr. Ramiro Martínez (Novozymes, Espanha) por gentilmente fornecer as enzimas usadas na pesquisa.

\section{REFERÊNCIAS}

BRADFORD, M.M. A rapid and sensitive method for the quantitation of microgram quantities of protein utilizing the principle of protein dye binding. Anal. Biochem, v.72, p. 248-254, 1976. 
DE MARIA, L.; VIND, J.; OXENBØLL, K.M.; SVENDSEN, A.; PATKAR, S;. Phospholipases and their industrial applications. Appl. Microbiol. Biotechnol. v.74, p.290-300, 2007.

FERNÁNDEZ-LORENTE, G.; PALOMO,J.M.; CABRERA, Z.; GUISÁN, J.M.; FERNÁNDEZLAFUENTE, R. Specificity enhancement towards hydrophobic substrates by immobilization of lipases by interfacial activation on hydrophobic supports. Enz. Microb. Technol., v. 41, p. 565-569, 2007.

GARCIA-GALANA, C.; SANTOS, J.C.S.; BARBOSA, O.; TORRES, R.; PEREIRA, E. B.; CORBERAN, V. C.; GONÇALVES, L.R.B.; FERNANDEZ-LAFUENTE, R. Tuning of Lecitase features via solid-phase chemical modification: Effect of the immobilization protocol. Process. Biochem., v.49, p. 604-616, 2014.

aSANTOS， J.C.S,; GARCIA-GALAN， C.; RODRIGUES， R.C.; SANTA'ANA， H.B.; GONÇALVES, L.R.B.; FERNANDEZ-LAFUENTE, R. Improving the catalytic properties of immobilized Lecitase via physical coating with ionic polymers. Enz. .Microb. Techn. v.60, p.1-8, 2014.

'bANTOS, J.C.S; GARCIA-GALAN, C.; RODRIGUES， R.C.; SANTA'ANA, H.B.; GONÇALVES, L.R.B.; FERNANDEZ-LAFUENTE, R. Stabilizing hyperactivated lecitase structures through physical treatment with ionic polymers.. Process. Biochem. manuscript accepted, 2014. 